\title{
Interfacial Structure-Modulated Plasmon-Induced Water Oxidation on Strontium Titanate
}

\author{
Xu Shi ${ }^{1 \#}$, Xiaowei Li ${ }^{2 \#}$, Takahiro Toda ${ }^{2}$, Tomoya Oshikiri ${ }^{1}$, Kosei Ueno ${ }^{2}$, Kentaro \\ Suzuki $^{2}$, Kei Murakoshi $^{2 *}$ and Hiroaki Misawa ${ }^{1,3 *}$
}

1. Research Institute for Electronic Science, Hokkaido University, Sapporo, Hokkaido 001-0021, Japan

2. Department of Chemistry, Faculty of Science, Hokkaido University, Sapporo, Hokkaido 060-0810, Japan

3. Center for Emergent Functional Matter Science, National Chiao Tung University, 1001 University Road, Hsinchu30010, Taiwan

\# Both authors contributed equally to this work.

*Corresponding authors: kei@sci.hokudai.ac.jp and misawa@es.hokudai.ac.jp 


\section{X-ray diffraction patterns of STO}

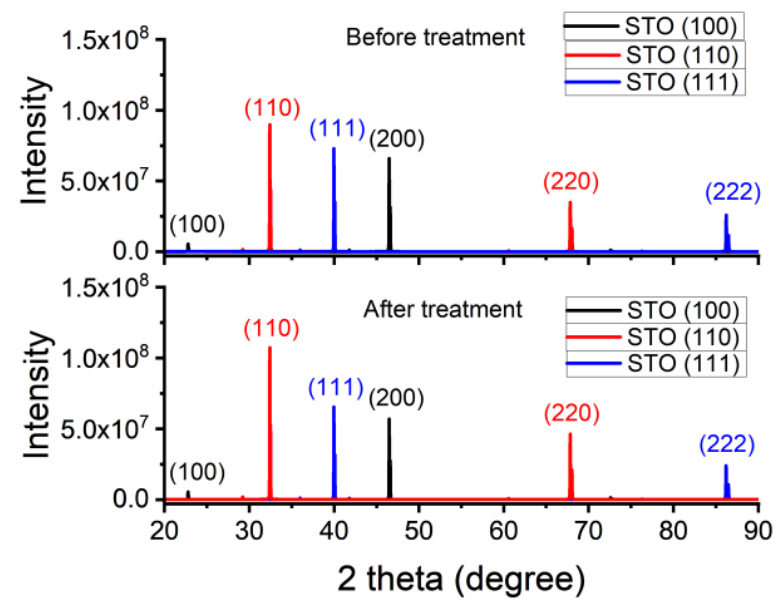

Figure S1. X-ray diffraction (XRD) patterns of STO with orientation of (100), (110) and (111) before and after experimental treatments. The XRD was performed on a Rigaku Smartlab with $\mathrm{Cu} \mathrm{K} \alpha$ source operating at $45 \mathrm{kV}$ and $200 \mathrm{~mA}$. The assignments of XRD peaks were noted in the figure.

\section{Size distribution of Au-NP}
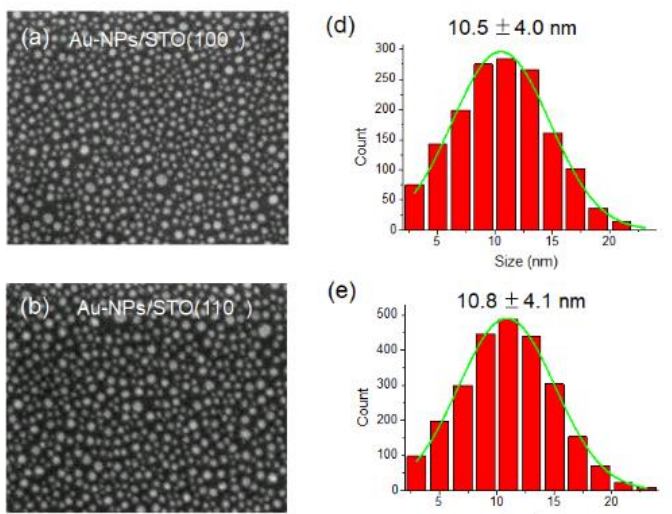

(e)
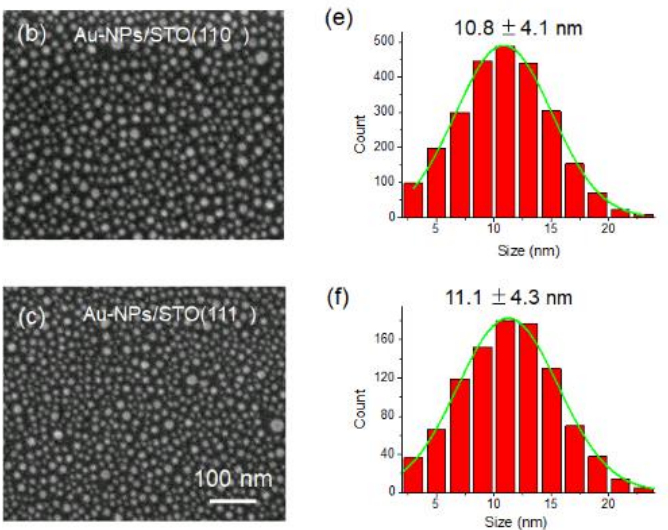

Figure S2. Surface morphology of Au-NP/STO. (a-c) The respective top view scanning electron microscopy (SEM) images of Au-NP loaded on STO (100), (110) and (111) surface, respectively. The scale-bar represents $100 \mathrm{~nm}$. (d-f) The respective size distribution analyzed from the SEM images. The sizes of Au-NPs on STO (100), (110) and (111) were estimated to be $10.5 \pm 4.0 \mathrm{~nm}$, $10.8 \pm 4.1 \mathrm{~nm}$ and $11.1 \pm 4.3 \mathrm{~nm}$, respectively. 


\section{Photoelectrochemical measurements}

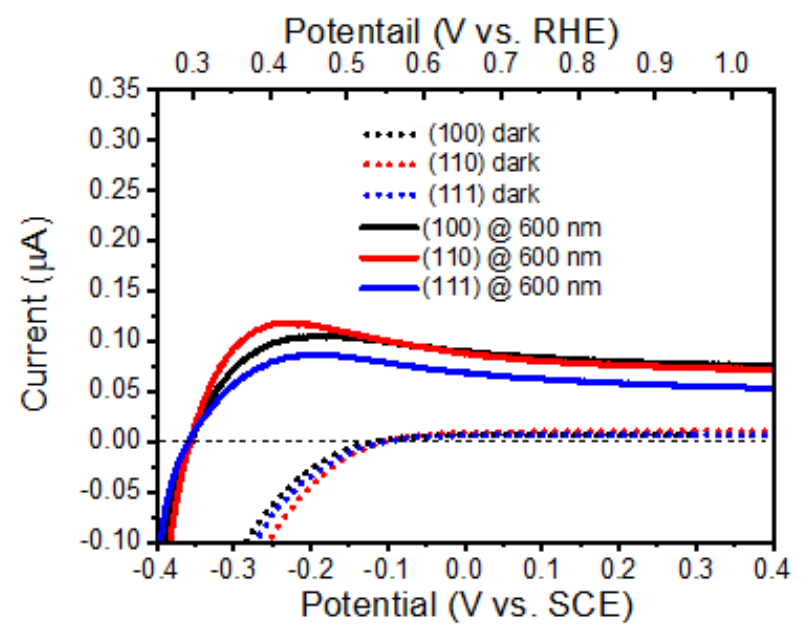

Figure S3. Current-potential curves of Au-NP/STO under dark (dashed lines) and $600 \mathrm{~nm}$ irradiation (solid lines). The scanning speed was $10 \mathrm{mV} / \mathrm{s}$.
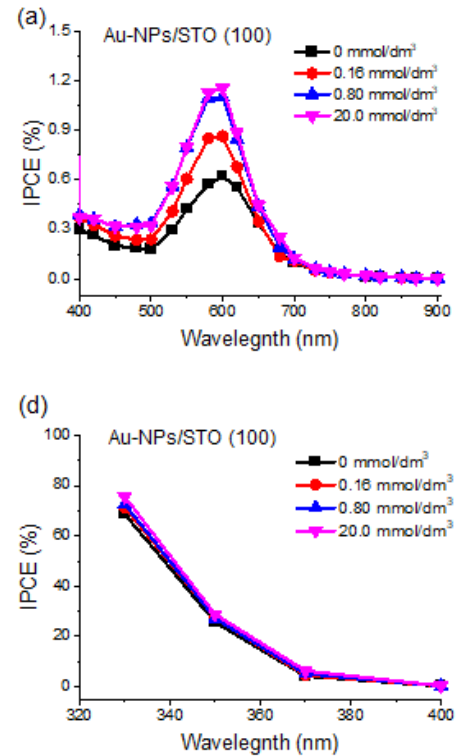
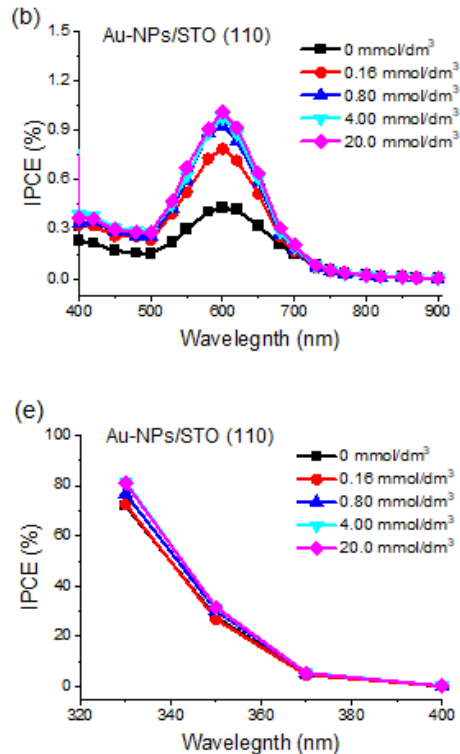
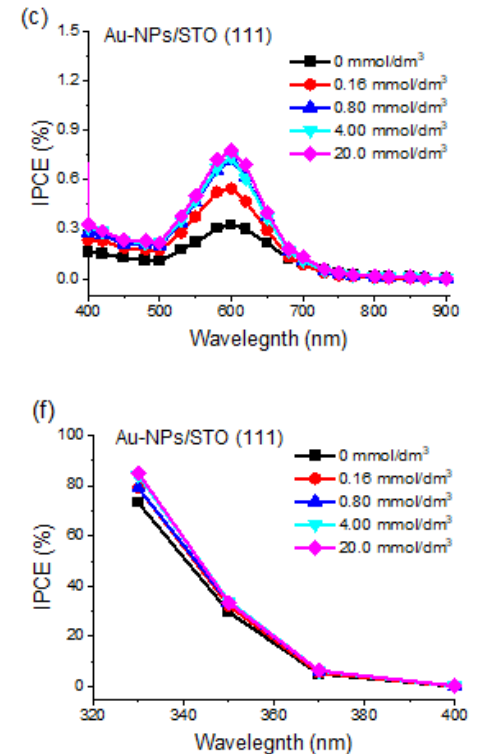

Figure S4. IPCE action spectra of Au-NP/STO with different STO orientated surface recorded at $0.3 \mathrm{~V}$ vs. SCE. (a-c) IPCE spectra of Au-NP/STO (100), Au-NP/STO (110) and Au-NP/STO (111) at the wavelength region of $400-900 \mathrm{~nm}$. The color curves represent electrolyte of $0.1 \mathrm{M}$ $\mathrm{KClO} 4$ with different concentration of triethanolamine (TEOA), from 0.16 to $20 \mathrm{mmol} / \mathrm{dm} 3$. (df) IPCE in UV region corresponding to (a-c). 


\section{Reference Raman spectra of $\mathrm{SrTiO}_{3}$}

In order to elucidate the electrochemical potential-dependent intermediates of $\mathrm{Au}$ surface oxidation progressing on (100) and (110) oriented STO substrate, Raman spectra of Au-NP/STO recorded at $-0.6 \mathrm{~V}$ vs. $\mathrm{Ag} / \mathrm{AgCl}(0 \mathrm{~V}$ vs. $\mathrm{RHE})$ were used as the reference spectra to obtain differential spectra. This potential is closed to the flat band potential of STO single crystal of $0.09 \mathrm{~V}$ vs. RHE estimated from the Mott-Schottky plot in Fig. $2 \mathrm{f}$ in the main text. $\mathrm{SrTiO}_{3}$ single crystal is paraelectric at all temperatures with centrosymmetric cubic perovskite structure $\mathrm{m} 3 \mathrm{~m}$ above $105 \mathrm{~K}$, and all the phonons are symmetry forbidden in the first-order Raman scattering. In Fig. S5, Raman bands appeared in the 200 400 $\mathrm{cm}^{-1}$ and $600 \sim 800 \mathrm{~cm}^{-1}$ range are assigned to typical second-order Raman spectra of STO in room temperature, as shown in Table S1.
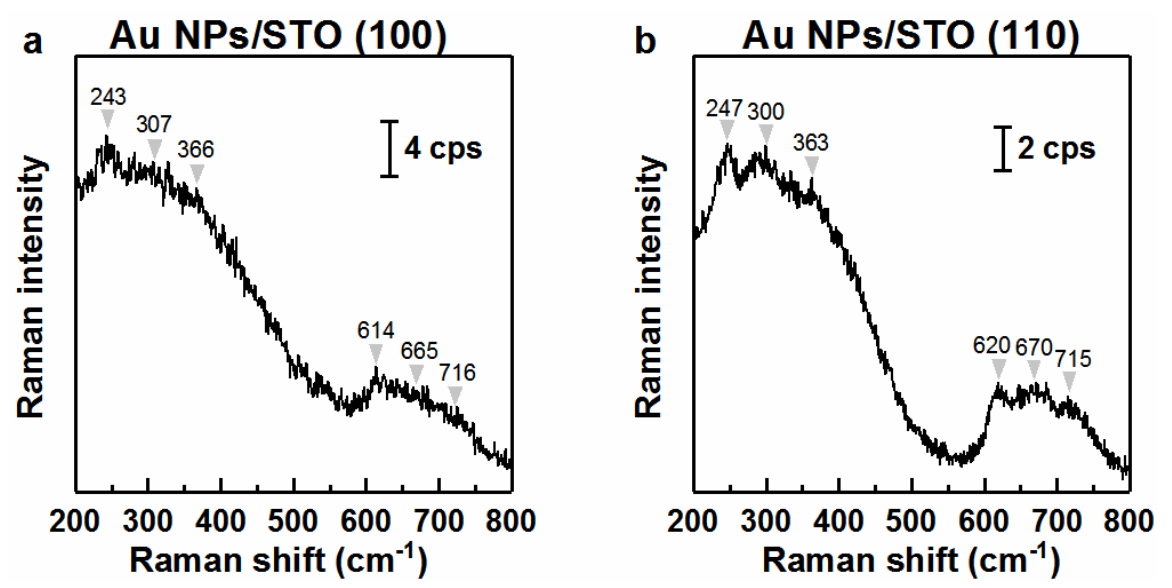

Figure S5. Raman spectra of $\mathrm{Au} \mathrm{NPs} / \mathrm{SrTiO}_{3}$ (perovskite) single crystal with (a) (100) and (b) (110) facet recorded at $-0.6 \mathrm{~V}$ vs. $\mathrm{Ag} / \mathrm{AgCl}(0 \mathrm{~V}$ vs. $\mathrm{RHE})$ in $0.1 \mathrm{M} \mathrm{NaF}(\mathrm{pH} \sim 7)$ electrolyte at room temperature. Laser wavelength of $785 \mathrm{~nm}$, laser power of $1.00 \mathrm{~mW}$, and acquisition time of $30 \mathrm{~s}$. Reverse triangles indicate the Raman shifts. 
Table S1 Phonon branch assignments for the second-order Raman spectra

\begin{tabular}{|llll|}
\hline Raman shift $/ \mathrm{cm}^{-1}$ & & Assignment & Two-phonon processes \\
$\mathrm{Au}-\mathrm{NP} / \mathrm{STO}(100)$ & $\mathrm{Au}-\mathrm{NP} / \mathrm{STO}(110)$ & & \\
\hline 243 & 247 & $2 \mathrm{TO}_{1}$ & Overtone \\
307 & & $\mathrm{TO}_{1}+\mathrm{TO}_{2}$ & \\
& 300 & $\mathrm{TA}^{2} \mathrm{TO}_{2}$ & Addition Combinations \\
366 & 363 & $\mathrm{LO}_{3}-\mathrm{TO}_{2}$ & Difference Combinations \\
\hline 614 & 620 & $\mathrm{LO}_{2}+\mathrm{TO}_{3}$ & \\
665 & 670 & $\mathrm{TO}_{1}+\mathrm{LO}_{3}$ & Addition Combinations \\
716 & 715 & $\mathrm{TO}_{4}+\mathrm{TO}_{2}$ & \\
\hline
\end{tabular}

There are four doubly degenerate transverse optic modes $\left(\mathrm{TO}_{1}, \mathrm{TO}_{2}, \mathrm{TO}_{3}\right.$, and $\left.\mathrm{TO}_{4}\right)$, four longitudinal optical modes $\left(\mathrm{LO}_{1}, \mathrm{LO}_{2}, \mathrm{LO}_{3}\right.$, and $\left.\mathrm{LO}_{4}\right)$, a doubly degenerate transverse acoustic mode (TA), aid a single longitudinal acoustic mode (LA) at the zone edge of STO. ${ }^{1}$

\section{Raman bands assignation on Au-NP/STO (100) and Au-NP/STO(110)}

The surface state of the STO (110) facet and dissipation of localized hot-holes are considered to contribute to the spectral features of the Au oxide intermediates. The plasmon-excited hotholes are confined around Au-NPs within several nanometers according to our previous visualized confirmation of polymerization reaction of pyrrole on $\mathrm{Au}-\mathrm{NPs} / \mathrm{TiO}_{2},{ }^{2}$ revealing that the water oxidation reaction occurs at this localized space on the interface between Au-NPs and STO. The local space occupied by confined hot-holes, however, is rather large around the random Au-NPs compared to the Au triangular nanodimers, indicating that certain number of hot-holes could dissipate onto the surface of STO and trap *OH that absorbed on Au-NPs (the asterisk denotes an adsorption site on the Au surface). Such communal ${ }^{*} \mathrm{OH}$ that linked both to Au and Ti or Sr of STO could result in inductive effect. The bonding electron cloud of Au-O deviates from oxygen atom nucleus, strengthens the Au-O bond and thus shifts the $v(\mathrm{Au}-\mathrm{O})$ mode to a higher frequency. ${ }^{3}$ And the adsorption amount of $* \mathrm{OH}$ on Au gradually raised as the electrochemical potential turned to positive, leading to enlarged Raman intensity and more obvious splitting spectral characteristics. One assumption that assigning the $481 \mathrm{~cm}^{-1}$ band to Ti-O or Sr-O stretching is ruled out for the reason that neither typical vibrations at $610 \mathrm{~cm}^{-1}\left(\mathrm{~A}_{1 \mathrm{~g}}\right)$ or $450 \mathrm{~cm}^{-1}$ $\left(\mathrm{E}_{\mathrm{g}}\right)$ originated from rutile $\mathrm{TiO}_{2}{ }^{4}$ nor two-photon assisted vibration at $520 \mathrm{~cm}^{-1}$ stemmed from $\mathrm{SrO}^{5}$ were observed in the resulted spectra, demonstrating that this $481 \mathrm{~cm}^{-1}$ band reasonably relates to the intermediate during Au oxidation and that the STO layer serves only as a support for hot-electron injection instead of a catalyst site for water oxidation. On the other respect, the fact that the $v(\mathrm{Au}-\mathrm{O})$ vibration on STO (100) surface, the macroscopic dipole perpendicular to the surface of the $\mathrm{STO}(110)$ facet due to the $\mathrm{SrO}^{4+}$ or $\mathrm{O}_{2}{ }^{4-}$ termination reasonably repulses the 
electron cloud of the oxygen atom in the Au-O bond, lengthen it to a certain extent and thus reduce the bond strength to a lower vibration frequency. In this sense, it is the more complicated surface condition of STO (110) that result in an alternative reaction process with intermediates by forming comparatively weak Au-O bonding and consequently reduce reactivity of plasmon-induced oxygen evolution reaction.
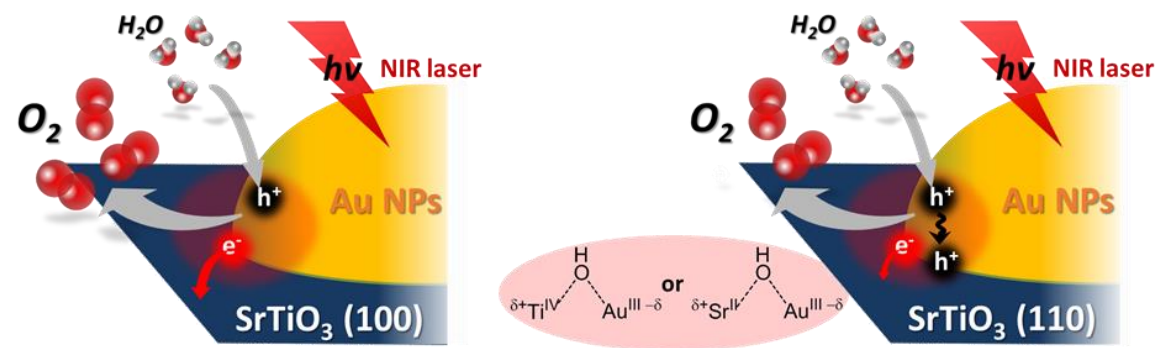

Figure S6. Schematic of plasmon-induced water oxidation around Au NPs loaded on (a) STO (100) and (b) (110) facet. Illustration in pink color presents possible absorption of $* \mathrm{OH}$ both on gold surface and on titanium or strontium atom of STO (110) facet.

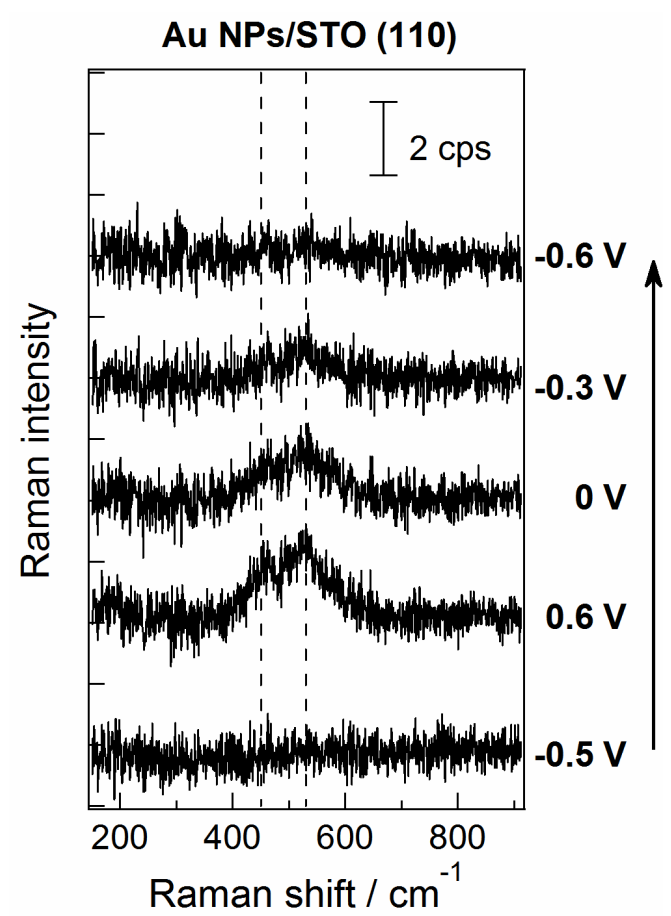

Figure S7. SERS spectra of Au NPs/STO (110) electrode in $0.1 \mathrm{M} \mathrm{NaF}$ prepared with $\mathrm{H}_{2}{ }^{18} \mathrm{O}$ recorded from $-0.5 \mathrm{~V}$ to $0.6 \mathrm{~V}$ and reversely to $-0.6 \mathrm{~V}$. The spectrum measured at flat band potential of $-0.6 \mathrm{~V}$ was used as the reference spectrum. Laser wavelength of $785 \mathrm{~nm}$, laser power of $1.00 \mathrm{~mW}$, acquisition time of $30 \mathrm{~s}$. All the potentials shown here are vs. $\mathrm{Ag} / \mathrm{AgCl}$. 

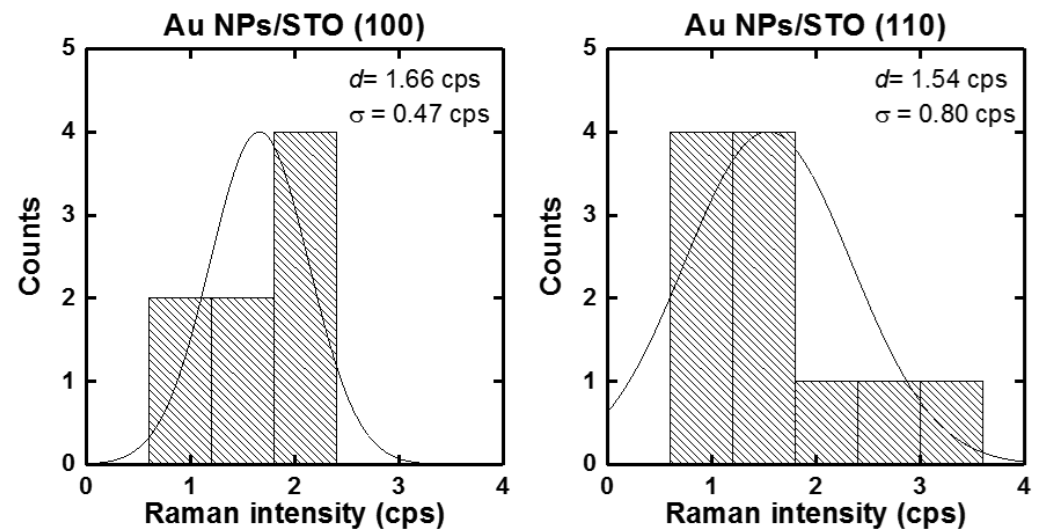

Figure S8. Raman intensity Distribution of Raman band at (left) $570 \mathrm{~cm}^{-1}$ for Au NPs/STO (100) and (right) $561 \mathrm{~cm}^{-1}$ for Au NPs/STO (110) electrode in $0.1 \mathrm{M} \mathrm{NaF}$ prepared with $\mathrm{H}_{2}{ }^{16} \mathrm{O}_{\text {or }} \mathrm{H}_{2}{ }^{18} \mathrm{O}$ recorded at $0.5 \mathrm{~V}$ vs. $\mathrm{Ag} / \mathrm{AgCl}$.

\section{IPCE enhancement}

According to the equation (2) in the main text, the rate of electron transfer from the redox molecule to the semiconductor is given by: ${ }^{6}$

$$
j=K^{\prime} \exp \left[-\frac{\left(E_{\text {redox }}^{0}-E_{s}+\lambda\right)^{2}}{4 k T \lambda}\right]
$$

where $K^{\prime}$ is the pre-exponential coefficient, $E_{S}$ indicates the electrochemical potential of the surface states, $E_{\text {redox }}^{0}$ is the electrochemical potential of the redox system, $\lambda$ is the reorganization energy. The pre-exponential coefficient $K^{\prime}$ is determined by the frequency of the reaction coordinate, the concentration of redox molecule and charge density at the surface. The $K^{\prime}$ equally increases in all Au-NP/STO after adding the TEOA. It is not the main contribution to the difference of IPCE on Au-NP/STO with different orientation surface of STO. For the specific chemical reaction, the reorganization energy $\lambda$ is constant, generally in the order of $1 \mathrm{eV}$, and the redox potential is the same for all Au-NP/STO. Therefore, the difference of IPCE on AuNP/STO with different orientation surface of STO might stems from the electrochemical potential difference of the surface states formed at the boundary of Au-NP/STO. The DFT calculation also shows that an interface electronic states are introduced in the bandgap (1.5-2.5 V vs. RHE) when settling a $\mathrm{Au}$ cluster on the surface of $\mathrm{TiO}_{2} .{ }^{7}$ The IPCE enhancement was the ratio between IPCE with and without TEOA. According to equation (1) and taking the value of $E_{T E O A}^{0}=1.1 \mathrm{~V}$ vs. RHE and $E_{\mathrm{H}_{2} \mathrm{O}}^{0}=1.23 \mathrm{~V}$ vs. RHE, the ratio is:

$$
\begin{gathered}
\mathrm{R}=\mathrm{j}_{\text {TEOA }} / \mathrm{j}_{\mathrm{H}_{2} \mathrm{O}} \\
\left.=K^{\prime \prime} \exp \left[-\left(1.11-E s+\lambda_{\mathrm{TEOA}}\right)^{2} / 4 k T \lambda_{\mathrm{TEOA}}+\left(1.23-E s+\lambda_{\mathrm{H}_{2} O}\right)^{2} / 4 k T \lambda_{\mathrm{H}_{2} \mathrm{O}}\right)\right]
\end{gathered}
$$


where $K^{\prime \prime}=K^{\prime}{ }_{T E O A} / K_{H_{2} O}^{\prime}$ is the kinetics enhancement of the chemical reaction, which is the same for all STO orientation surface. The only variable is equation (2) is $E_{s}$, the energy level of surface states, and it is possible to qualitatively calculate the relationship between $R$ and $E_{s}$, as shown in Fig. S9. It shows that the IPCE enhancement decreases as the energy level of the surface states increases.

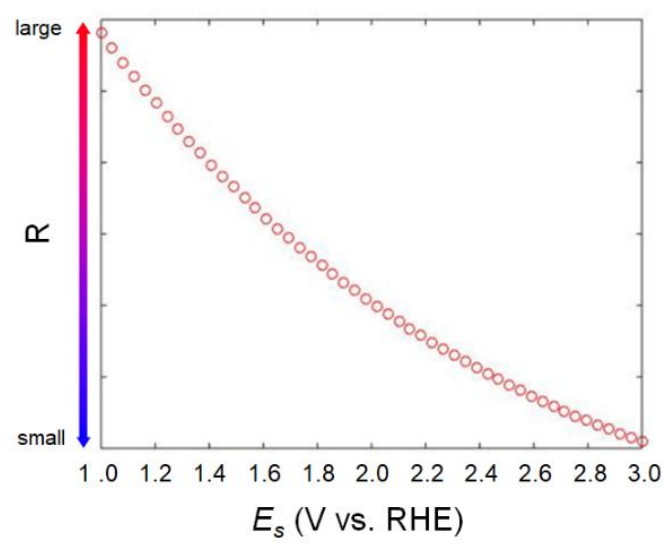

Figure S9. Qualitative calculation of the relationship between the IPCE enhancement, R, and the energy level of the surface states, $E_{s}$.

\section{References}

1. Perry, C. H.; Fertel, J. H.; McNelly, T. F. Temperature Dependence of the Raman Spectrum of $\mathrm{SrTiO}_{3}$ and $\mathrm{KTaO}_{3}$, J. Chem. Phys. 1967, 47, 1619.

2. Minamimoto H.; Toda T.; Futashima R.; Li X.; Suzuki K.; Yasuda S.; and Murakoshi K., Visualization of Active Sites for Plasmon-Induced Electron Transfer Reactions Using Photoelectrochemical Polymerization of Pyrrole, J. Phys. Chem. C 2016, 120, 16051-16058

3. Nakamoto, K., Infrared and Raman Spectra of Inorganic and Coordination Compounds. In Handbook of Vibrational Spectroscopy, Wiley, New York (2006).

4. Arsov, L. D.; Kormann, C.; Plieth, W., Electrochemical synthesis and in situ Raman spectroscopy of thin films of titanium dioxide. J. Raman Spectrosc. 1991,22, 573-575.

5. de Waal, D.; Range, K. J.; Königstein, M.; Kiefer, W., Raman spectra of the barium oxide peroxide and strontium oxide peroxide series. J. Raman Spectrosc. 1998, 29, 109-113.

6. Memming, R. Semiconductor Electrochemistry, 2nd ed.; Wiley-VCH: Weinheim, Germany, 2015.

7. Wang, S.; Gao, Y.; Miao, S.; Liu, T.; Mu, L.; Li, R.; Fan, F.; Li, C., Positioning the Water Oxidation Reaction Sites in Plasmonic Photocatalysts. J. Am. Chem. Soc. 2017, 139, 11771-11778. 